\title{
Lateral Migration Radiography
}

\author{
E. T. Dugan, ${ }^{1}$ A. M. Jacobs, ${ }^{1}$ S. P. Keshavmurthy, ${ }^{2}$ J. C. Wehlburg ${ }^{3}$ \\ ${ }^{1}$ Department of Nuclear and Radiological Engineering, University of Florida, Post Office Box 118300, \\ Gainesville, FL 32611-8300, USA \\ ${ }^{2}$ Department of Radiology, University of Michigan, 1500 East Medical Center Drive, Ann Arbor, MI \\ 48109, USA \\ ${ }^{3}$ Sandia National Laboratory, Post Office Box 5800, MS 1182, Albuquerque, NM 87185, USA
}

\begin{abstract}
Lateral migration radiography (LMR) is a new form of Compton backscatter imaging (CBI) that utilizes both multiple-scatter and single-scatter photons. The LMR imaging modality uses two pairs of detectors. Each set has a detector that is uncollimated to predominantly image single-scatter photons and the other collimated to image predominantly multiple-scattered photons. This allows generation of two separate images, one containing primarily surface features and the other containing primarily subsurface features. These two images make LMR useful for imaging and identifying objects to a depth of several X-ray photon mean free paths even in the presence of unknown surface clutter or surface imperfections.

The principles of LMR are demonstrated through Monte Carlo simulation of the photon transport. The Monte Carlo simulation results are verified with experimental measurements from an LMR system used for landmine detection. The presented research demonstrates the methodology for designing an LMR system, identifies methods for restoring and enhancing LMR images, and lays the foundation for the development of other applications of LMR, including, for example, the nondestructive examination of welds, castings, and composites.
\end{abstract}

\section{Introduction}

Lateral migration radiography (LMR) is a new form of Compton backscatter imaging (CBI) that utilizes both multiple-scatter and single-scatter photons. This research develops the theory of LMR, examines its application to landmine detection, demonstrates the methodology for designing an LMR system, presents methods for restoring and enhancing LMR images, and lays the foundation for the development of other applications of LMR, such as the nondestructive examination of welds, castings, and composites.

A CBI system operates somewhat like an optical visual system, where the reflected photons from the surfaces of the objects are converted into an electrical signal and processed to form an image. The CBI technique using X-rays has been applied successfully to commercial products such as airport luggage scanning systems [1], COMSCAN [2], tomography systems, and developmental systems for the functional imaging of the 
heart [3]. There are other CBI applications such as COMTEL (Compton telescope) [4], void fraction measurement in two-phase fluids [5], and noninvasive evaluation of coronary bypass grafts that use the spectral analysis of the scattered photons to image objects [6]. All of the above-mentioned CBI systems rely on first-scattered photons from the object to form images. Surface imperfections and ridges affect the CBI image quality because these features can prevent first-collided photons from reaching the detectors and lead to multiple-collision photons, which now enter the detectors. Because of this obstruction and corruption, the CBI images of an object with unknown surface imperfections do not uniquely represent the surface and the regions underneath. Hence, slow running complex algorithms are necessary to extract useful information. Additionally, the dependence of CBI systems on first-collision backscatter photons to form images also imposes a constraint on detector size, collimation, and mode of detector operation. This limitation often leads to high source strength and slow imaging system operation. LMR is a new imaging modality developed at the University of Florida [7-10]. As the name suggests, this modality uses the lateral transport of multiple-scattered photons in materials to form images. Large area detectors operating in the current or integration mode rather than in the voltage or counting mode help to reduce the required X-ray source strength and image acquisition time. LMR systems typically use two sets of detectors to form images. The first set of uncollimated detectors image predominantly first-collision photons, and a second set is collimated and placed so as to image predominantly multiple-collision photons. The uncollimated detectors primarily generate images of the surface features. The contrast in the collimated detector images is due to multiple-scattered photon lateral transport, which is sensitive to the electron density of the transport medium as well as the surface spatial details. This enables us to image objects that contain clusters of subtle imperfections and discontinuities in electron density and identify such electron density differences. The multiple-collision photons always carry the information from the first collision. However, with the increase in the number of collisions, multiple-collision components average out small-sized electron density variations while retaining the information from the large-sized discontinuities. Because the LMR images are no longer restricted to first-scatter photons, this modality is useful for imaging objects even in the presence of surface clutter.

This research studies the details of photon transport in an LMR system using the Monte Carlo technique and explains both qualitatively and quantitatively the reasons for the image contrast and features due to lateral migration. The Monte Carlo results are verified with measurements from an LMR system used for landmine detection. On the basis of the detailed Monte Carlo analysis, two approximate solutions to photon transport in the LMR systems are developed. The first approach approximates the solution by treating the LMR image formation as a pseudoinverse transport problem and leads to an LMR image restoration algorithm and a method of predicting buried landmine parameters (mine radius, depth of burial, and $x-y$ location). The second method approximates the photon transport with a specialized, fast-running Monte Carlo-based lateral transport simulation algorithm. The results from these two solution methods are compared with measured results. Results from the LMR photon transport study are used to predict optimum X-ray source and detector specifications for a representative LMR landmine detection system, and these predictions are also verified with measurements. 


\section{Scatter Radiography}

As the name suggests, CBI relies on Compton scatter to generate images. In simple terms, Compton scatter can be understood as a relativistic billiard ball collision between incident photons and the electrons after which the photon energy and angle change. The energy of the scattered photon is given by

$$
\alpha^{\prime}=\frac{\alpha}{1+\alpha(1-\mu)},
$$

where $\alpha$ and $\alpha^{\prime}$ are the incident and scattered photon energies, respectively, expressed in terms of electron rest mass, and $\mu$ is the cosine of the scattering angle. The Klein-Nishina differential scattering cross section gives the probability of scatter from free electrons in a given direction as

$$
\sigma(\alpha, \mu) d \mu=\pi r_{0}^{2}\left(\frac{\alpha^{\prime}}{\alpha}\right)^{2}\left(\frac{\alpha^{\prime}}{\alpha}+\frac{\alpha}{\alpha^{\prime}}+\mu^{2}-1\right) d \mu \quad\left(\mathrm{cm}^{2} / \text { electron }\right)
$$

where $r_{0}$ is the classical electron radius. The above two equations form the basis for defining Compton scatter from a material. The scattering probability from a material is given by

$$
P_{s}=\frac{\sigma_{s}}{\sigma_{t}}=\frac{\sigma_{s}}{\sigma_{a}+\sigma_{s}}
$$

where $\sigma_{s}$ is the scattering cross section and $\sigma_{t}$ is the total cross section of the material. The total cross section is the sum of the absorption cross section $\sigma_{a}$ and the scattering cross section. $\sigma_{a}$ is the sum of the photoelectric cross section, which is high at very low energies, and the pair production cross section, which increases with the photon energy above the 1.02-MeV threshold. Both the photoelectric and pair production cross sections increase as $Z^{n}$, where $n$ is greater than 1 . However, the scattering cross section $\sigma_{s}$ increases only as $Z$, and it decreases with photon energy. Thus, from equation (3) it can be said that $P_{s}$ is higher for low- $Z$ materials than for high- $Z$ materials.

As an example, $P_{s}$ for a typical soil $(Z=11)$ and a typical plastic landmine (TNT with $Z=7$ ) are compared in Fig. 1. It is clear from this figure that for the energy range of $20-200 \mathrm{keV}$, the $P_{s}$ value for mine (a low- $Z$ material) is greater than for soil. The $P_{s}$ for steel $(Z=26)$ is lower than that of soil and mine for the energy range between 20 and $200 \mathrm{keV}$. Using this sensitivity of photon scatter to $Z$, many imaging applications of Compton scatter have been developed. These applications are generally referred to as scatter radiography in the literature.

A schematic of the experimental setup used for the landmine detection measurements is shown in Fig. 2. This system uses a pair of uncollimated detectors and a pair of collimated detectors to generate four images of an object. The higher $P_{s}$ for low- $Z$ material is due to a relatively lower $\sigma_{a}$, which makes the mean free path (m.f.p.) of scatter photons larger in low- $Z$ regions than in high- $Z$ regions (the m.f.p. is also larger for low-density materials than for high-density materials). This difference in the photon travel path causes more multiple-scattered photons to reach the collimated detectors 


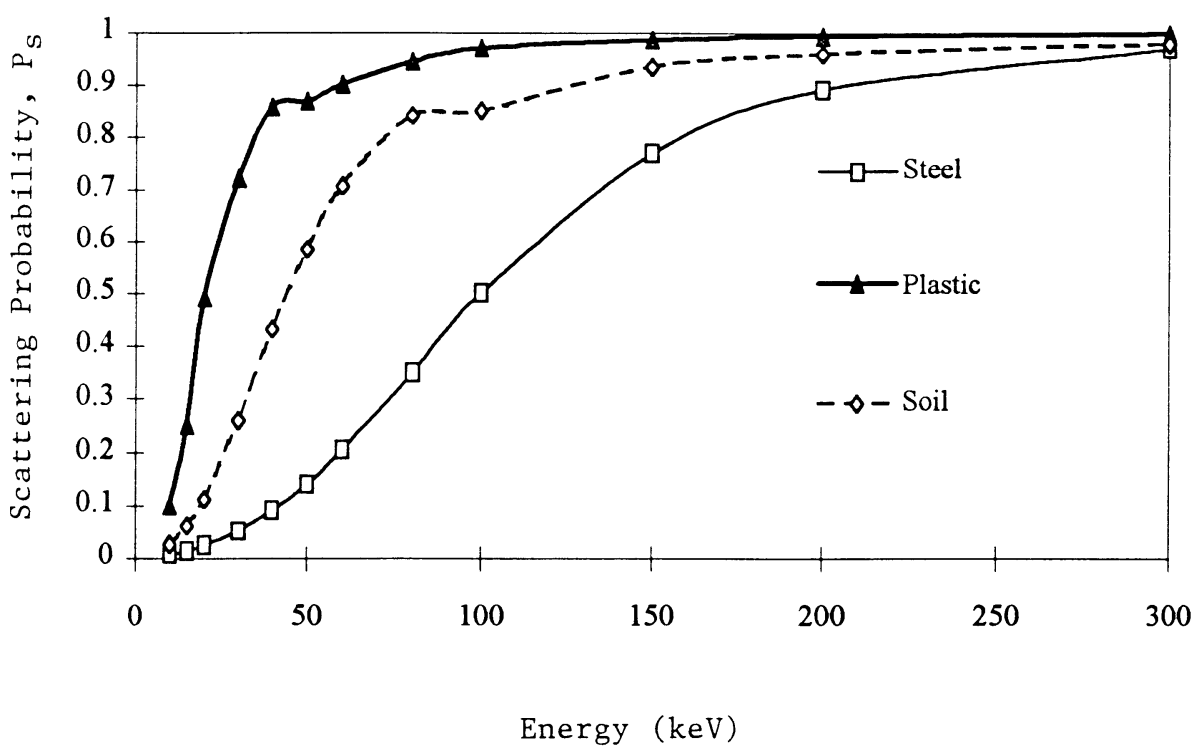

Fig. 1. Comparison of scattering probabilities of three materials.

from lower electron density regions than from higher electron density regions. Because of this difference, the collimated detector energy deposition is higher when the source is over a lower electron density region. The difference in detector energy deposition is observed as image contrast in LMR images that can be used to identify low electron density regions in a higher electron density medium (or vice versa). The utility of such a system to detect buried landmines in soil has been proven and with slight modifications can be used for the nondestructive examination of welds and castings (up to multi-mean free $X$-ray path depths) and thick, low- $Z$ materials like composites with subtle electron density differences.

\section{Monte Carlo Simulation of Photon Transport in the Landmine Imaging System with MCNP}

There are many commercially available Monte Carlo codes that handle particle transport and provide various options for variance reduction and for tallying particle histories. One of the most widely used and extensively tested Monte Carlo codes for neutron and/or photon transport is the MCNP code [11]. General-purpose Monte Carlo codes like MCNP provide flexibility in geometry description, source description, and material definition. Because of this flexibility, its long history of testing and development, and its rich variance reduction schemes, MCNP was chosen to simulate the photon transport for the study of lateral migration imaging in the buried landmine detection problem. Deterministic methods could be applied to the modeling of the LMR photon transport (e.g., a threedimensional discrete ordinates solution of the Boltzmann transport equation) but they 


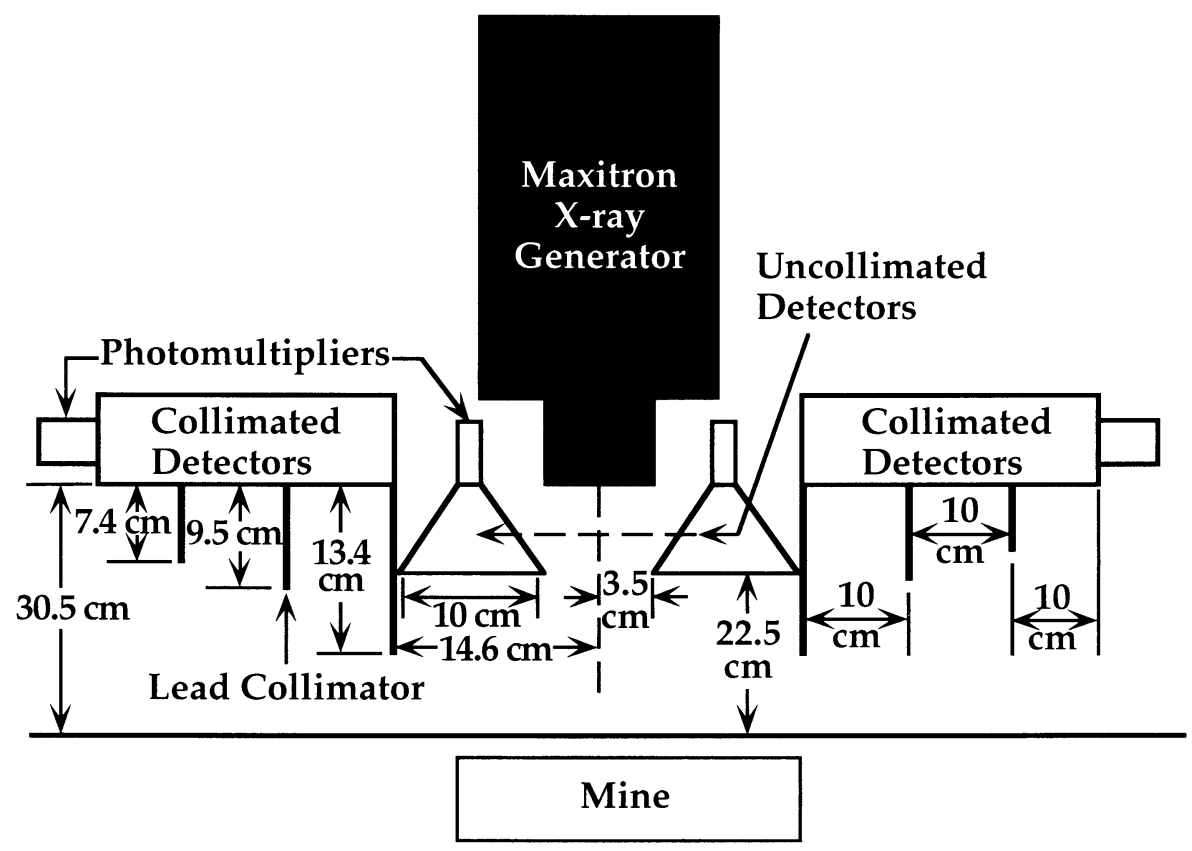

Fig. 2. Schematic of experimental setup used for the LMR landmine detection measurements.

would be essentially as time-consuming as the Monte Carlo approach and would not provide the detailed information required for an understanding of the physics of the lateral migration and LMR image formation processes.

The landmine imaging system consists of a pair of uncollimated detectors placed near the source beam and another pair of detectors with lead collimators located adjacent to these uncollimated detectors but farther from the X-ray beam (see Fig. 2). To generate an image the X-ray beam rasters in the gap between the two uncollimated detectors and also moves with the detectors in a direction orthogonal to this raster direction. The two beam travel directions are called the raster and motion directions, respectively, and the gap between the two uncollimated detectors is called the raster gap. However, in the experimental measurement system (see Fig. 3), the X-ray beam remains stationary and the soil box $(122 \mathrm{~cm} \times 141 \mathrm{~cm} \times 30.5 \mathrm{~cm})$ in which the mines are buried moves in the two orthogonal directions. This type of ground illumination is necessitated in the experimental measurements because of the constraints related to the available X-ray generator.

The uncollimated detectors modeled in the MCNP simulations are $140 \mathrm{~cm}$ long and $10 \mathrm{~cm}$ wide, whereas the detectors used in the experimental measurements are $30 \mathrm{~cm}$ long and $10 \mathrm{~cm}$ wide. The collimated detectors in the MCNP simulation are $140 \mathrm{~cm}$ long and $30 \mathrm{~cm}$ wide but $30 \mathrm{~cm}$ long and $30 \mathrm{~cm}$ wide in the experimental measurements. The length is measured along the raster direction and the width is measured along the motion direction. The difference in the detector size between the MCNP simulation model and 


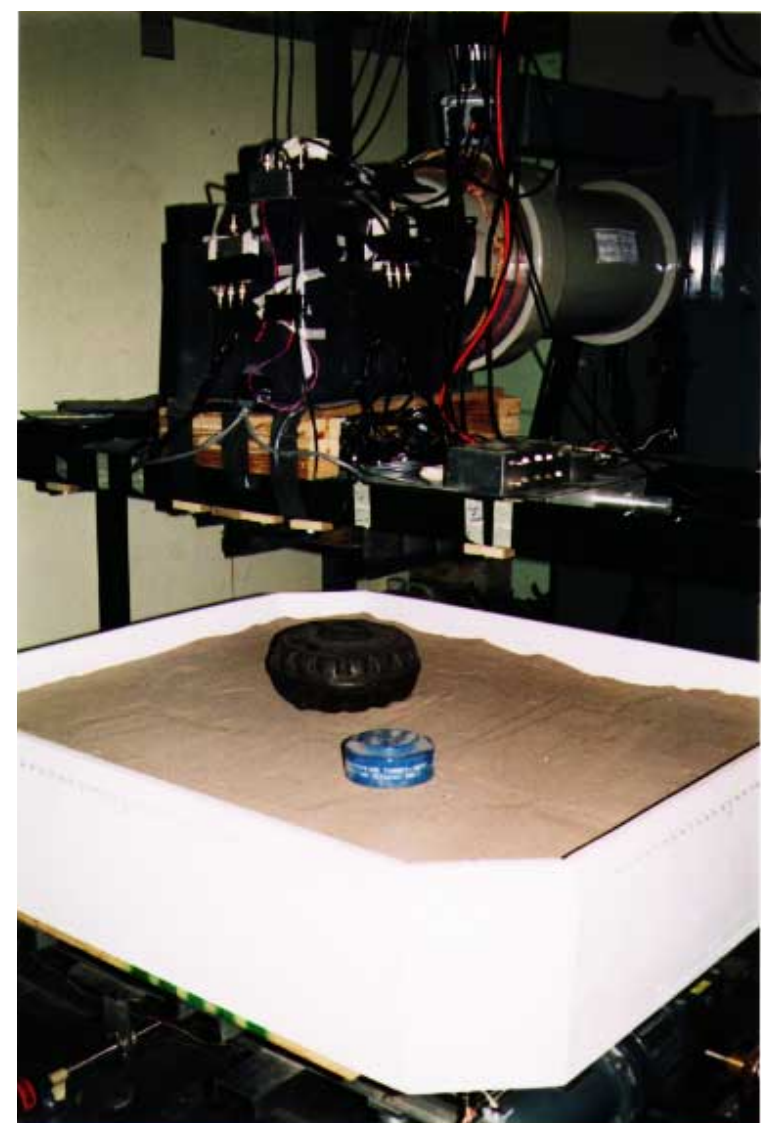

Fig. 3. Photograph of the experimental LMR landmine detection system.

the experimental system is due to the difference in the X-ray beam motion between the simulation and the measurement. It is important to note that the detector system motion modeled in the MCNP simulation is closer to that of an actual LMR landmine imaging system than the setup used for the presented experimental measurements. This is because in both the MCNP simulation and an actual LMR landmine detection system, the detectors move only in the motion direction with a vehicle, while the X-ray beam moves both in the vehicle motion direction and rasters orthogonal to this direction within the raster gap. This gives rise to an edge effect or detector signal fall-off as the source is moved in the raster direction toward the extreme end positions of the detectors. In contrast, in the experimental measurements, the soil box motion is used to simulate the X-ray beam motion in both the vehicle motion and raster directions. In so doing, motion also is simulated for the detectors in both the motion and the raster directions. The source and the detector locations are thus always fixed relative to each other instead of experiencing a relative change in position in the raster direction. The measurements therefore do not exhibit edge effects or detector signal fall-off in the raster direction. 


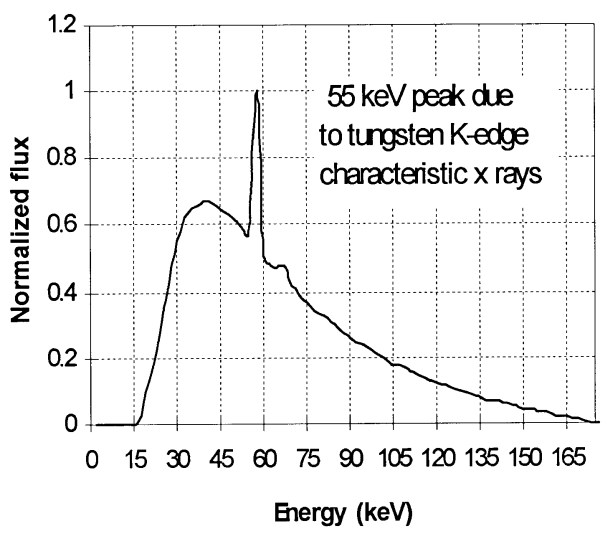

Fig. 4. $175-\mathrm{kVp}$ X-ray spectrum used for MCNP Monte Carlo photon transport simultions.

MCNP simulations were performed for a $30.5-\mathrm{cm}$ (12-in) diameter plastic landmine with a $2.5-\mathrm{cm}$ (1-in) depth-of-burial (DOB) in soil. The X-ray generator source strength was $175 \mathrm{kVp}$, and the source spectrum used for the simulation is presented in Fig. 4. The image size is 33 by 33 with $2.5-\mathrm{cm}$ pixels. All images presented in this paper, unless noted otherwise, used a $175-\mathrm{kVp}$ X-ray source and $2.5 \mathrm{~cm}$ by $2.5 \mathrm{~cm}$ pixels. The MCNP simulation used 300,000 source particles per pixel. The uncertainties in the energy deposition in the uncollimated and collimated detectors are 3.5 and $10 \%$, respectively. The collimated detector images generated by MCNP and by measurement are presented in Figs. 5 and 6, respectively. The uncollimated detector images obtained from MCNP and measurement are shown in Figs. 7 and 8, respectively.

The lines seen in the MCNP collimated and uncollimated detector images along the motion direction are due to the detector signal fall-off in the raster direction. This fall-off can be attributed to the reduction in the detector area seen by the scattered X-rays at the end pixel positions in the raster direction.

The front collimated detector images show a lateral shift toward the rear in the motion direction while the rear detectors show a shift toward the front. The actual mine center was at pixel positions 16 and 17 in the motion direction for the measurements and MCNP simulations, respectively. In the measured collimated detector images, the peaks are shifted to pixel positions 18 and 13 in the motion direction for the rear and the front detectors, respectively. Similarly, the peaks are shifted to pixel positions 19 and 14 in the MCNP images for the rear and the front detectors, respectively. The reason for this shift is presented in the next section. The uncollimated detector images from the MCNP simulations do not show any significant shift either in the motion or the raster direction, and the center of the mine is at pixel position $(17,17)$ in the simulation. The shift observed in the measured uncollimated detector images toward the rear in the motion direction is due to a slight slope that existed in the soil which caused the detector signals to be biased toward the higher side of the soil slope. Examination of many MCNP simulations for plastic and metal mines of different shapes and sizes (ranging from $7.6 \mathrm{~cm}$ in diameter to 


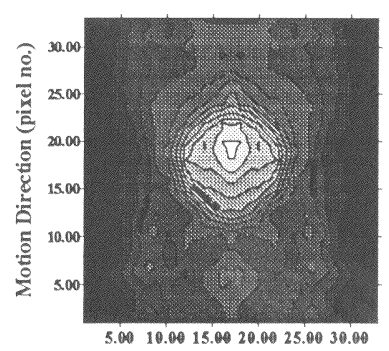

Raster Direction (pixel no.)

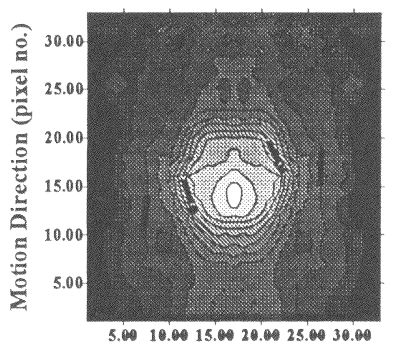

Raster Direction (pixel no.)

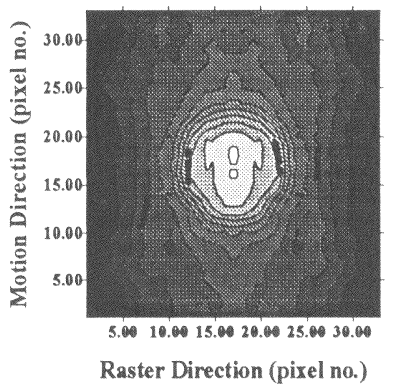

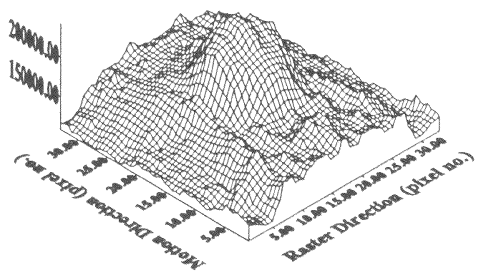

Rear Detector Image

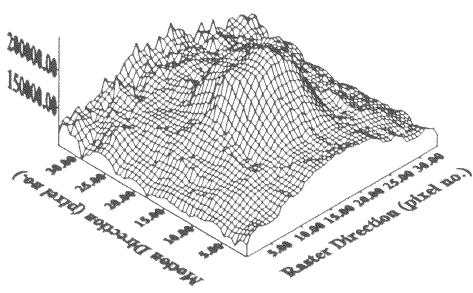

Front Detector Image

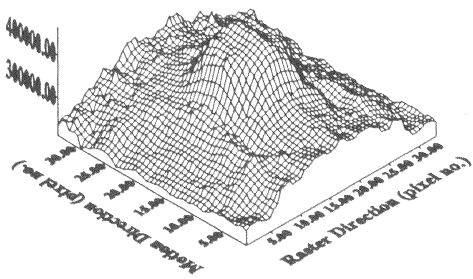

Sum of Two Detector Images

Fig. 5. The MCNP-generated collimated detector images of a 30.5-cm-diameter plastic landmine at pixel $(17,17)$ with a $2.5-\mathrm{cm}$ DOB and 2.5 -cm pixels.

$30.5 \mathrm{~cm}$ in diameter), mine DOBs from 0 to $7.6 \mathrm{~cm}$, and different soil environments has shown that the MCNP results are in agreement with measurements from the landmine imaging system and that MCNP can be used to analyze the details of the behavior of lateral migration imaging. 


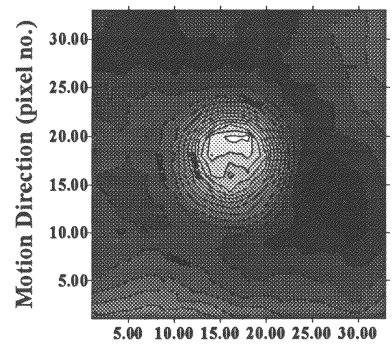

Raster Direction (pixel no.)

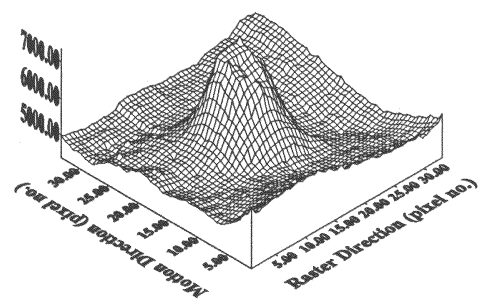

Rear Detector Image

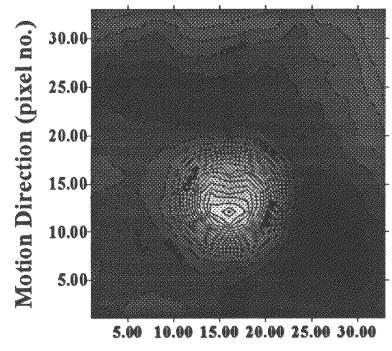

Raster Direction (pixel no.)

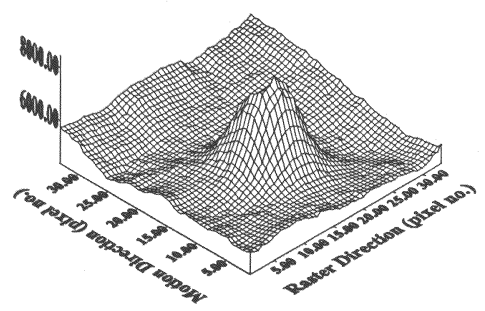

Front Detector Image
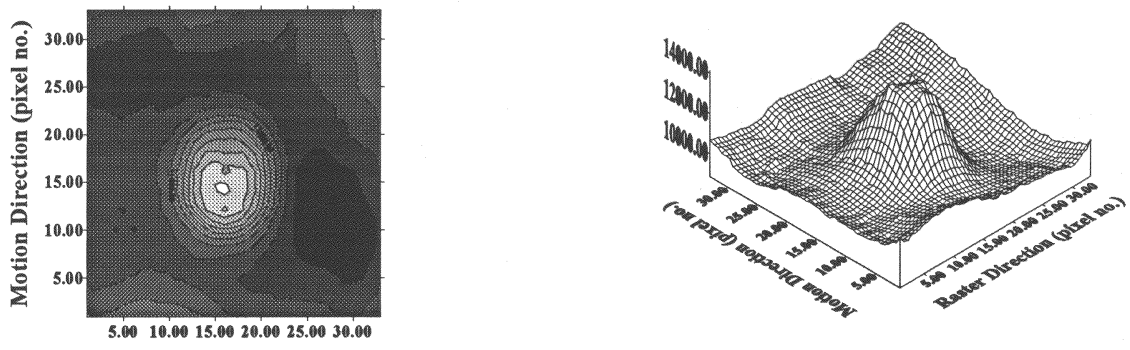

Raster Direction (pixel no.)

Sum of Two Detector Images

Fig. 6. The measured experimental collimated detector images of a 30.5-cm-diameter plastic landmine at pixel $(16,16)$ with a $2.5-\mathrm{cm}$ DOB and $2.5-\mathrm{cm}$ pixels.

\section{Photon Transport in an LMR System}

The general-purpose MCNP Monte Carlo code along with the photon track visualization code SABRINA [12] have been used to study photon transport in the LMR system used 

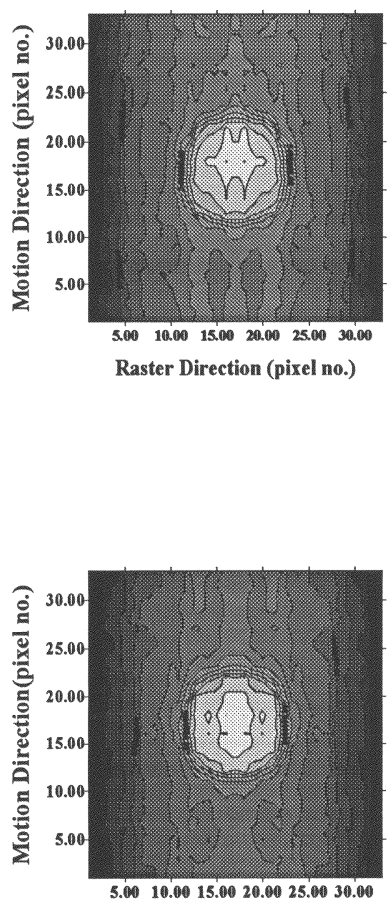

Raster Direction (pixel no.)

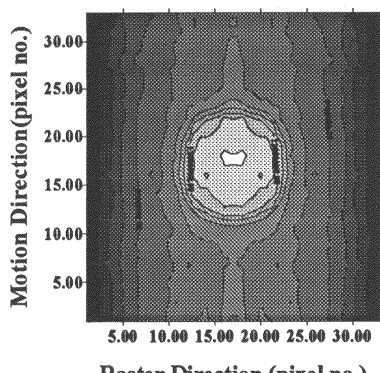

Raster Direction (pixel no.)

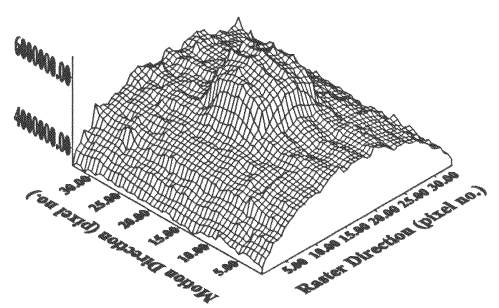

Front Detector Image

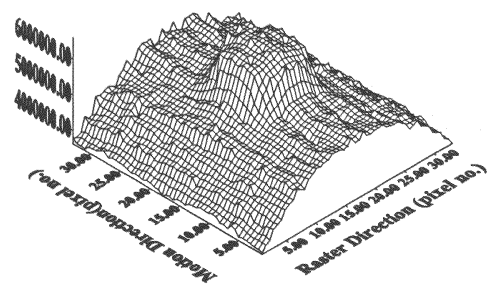

Rear Detector Image

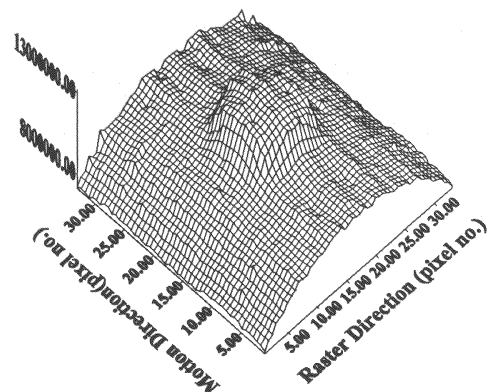

Sum of Two Detector Images

Fig. 7. The MCNP-generated uncollimated detector images of a 30.5-cm-diameter plastic landmine at pixel $(17,17)$ with a 2.5 -cm DOB and 2.5 -cm pixels.

for landmine detection. SABRINA plots results from an MCNP Monte Carlo simulation with tracks representing particle transport paths. These tracks can be superimposed on the simulation geometry so that the effect of each transport medium can be studied.

To study the photon transport in an LMR system the simulation of the landmine 

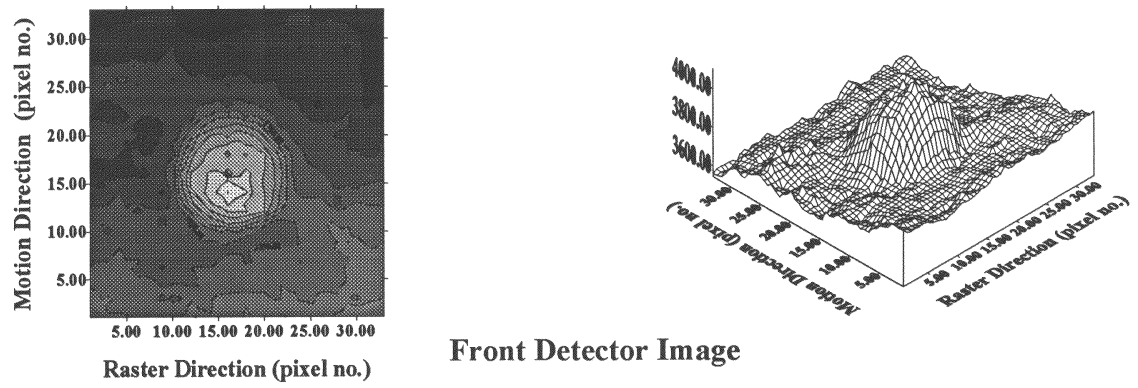

Front Detector Image
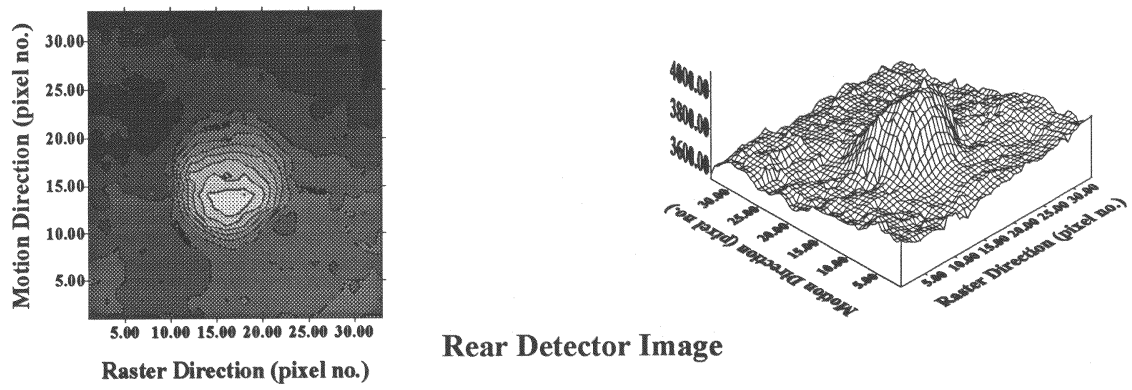

Rear Detector Image

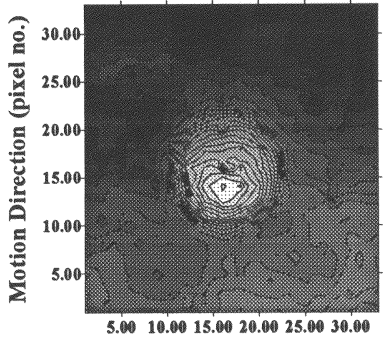

Raster Direction (pixel no.)

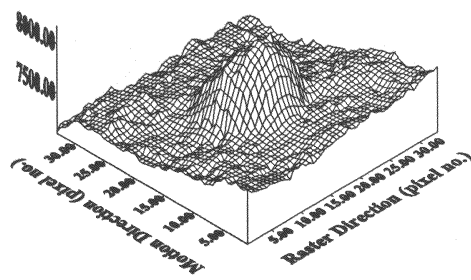

Sum of Two Detector Images

Fig. 8. The measured experimental uncollimated detector images of a $30.5-\mathrm{cm}$-diameter plastic landmine at pixel $(16,16)$ with a $2.5-\mathrm{cm}$ DOB and $2.5-\mathrm{cm}$ pixels.

detection system is carried out for a case with the mine at a DOB of $2.5 \mathrm{~cm}$ and the source positioned over the mine center. The MCNP simulation uses 200,000 source particles, out of which the first 30,000 particle events are logged. This results in a total of over 14,500 histories.

The results from the SABRINA simulation of the landmine imaging system are pre- 
sented graphically as the plots of photon tracks. The number of tracks to be plotted was chosen such that they not crowd the track plot too much but represent the trends of quantitative results predicted by MCNP. Figures $9 \mathrm{a}$ and $9 \mathrm{~b}$ present the first-collision tracks from both the mine and soil and from the mine only, respectively. The plots represent 13671 histories/31203 tracks and 1200 histories/2739 tracks, respectively. These results are from the same simulation and show that only about $10 \%$ of the first-scattered photons come from the mine. Figure 9a shows the effect of collimators in reducing the first-collision energy deposition in the collimated detectors; the energy deposition in the collimated detectors is about an order of magnitude less than that of the uncollimated detectors. Figure 9a also shows that the majority of the first-scatter photons originate from the surface and, hence, represent the surface features. The second-collision component tracks are presented in Fig. 10a. This figure represents the scatters from the mine and shows 1159 histories/6340 tracks. There are essentially no second-scatter photons between 170 and $180^{\circ}$. Additionally, most of the scatter photons are distributed very close to the incident beam and do not show any significant lateral transport in the mine.

The third- and fourth-collision component track plots are presented in Figs. 10b and 10c, respectively. These plots represent 1155 histories/5957 tracks and 1168 histories/6099 tracks, respectively. Unlike first- and second-scatter photons, these components show distinct lateral transport in the mine, and these laterally transported components give rise to a secondary X-ray spot in the mine. The collimated detector images are dominated by the high-collision components whereas the uncollimated detector images are dominated by the first-collision components. This imaging modality is referred to as lateral migration radiography because the image contrast in the collimated detectors is generated by a higher lateral migration of the multiple-scattered photons in a low- $Z$ medium. The lateral migration here is an enhanced transport of photons that occurs in low- $Z$ or lower density media (such as plastic or voids) or a reduced transport that occurs in high- $Z$ or higher density material (like metal) below the soil surface along directional components parallel to the soil surface.

To verify the observed angular distribution of scatter components and to calculate the scattering probabilities of Compton-scattered photons, the Klein-Nishina $(\mathrm{K}-\mathrm{N})$ cross-section relations are applied. On the basis of these $\mathrm{K}-\mathrm{N}$ scattering probabilities the average values of energy and scattering angles for scattered photons are calculated. The $\mathrm{K}-\mathrm{N}$ scattering cross sections are calculated first for forward and backscattered photons. These calculated cross sections then are used to evaluate the average scattering angles and energies.

The results for the first Compton scatter are presented in Table 1. The average energy and scattering angle for forward- and backscattered photons are given as a function of incident photon energy. The difference in the energies of forward- and backscatter photons increases with an increase in the source energy. However, it can be seen from Table 1 that for the source energy between 40 and $100 \mathrm{keV}$, the average energy of the forward- and backscattered photons are fairly similar. This energy range plays a very important role in LMR landmine detection because the average source energy for $120-200-k$ Vp X-ray spectra is about $40-100 \mathrm{keV}$, and the optimum X-ray source energy for the detection of buried mines in typical soils is $175-185 \mathrm{kVp}$ for an AC X-ray source and $125-130 \mathrm{kVp}$ for a DC source.

It can be seen from Table 1 that for a monoenergetic source in the energy range between 


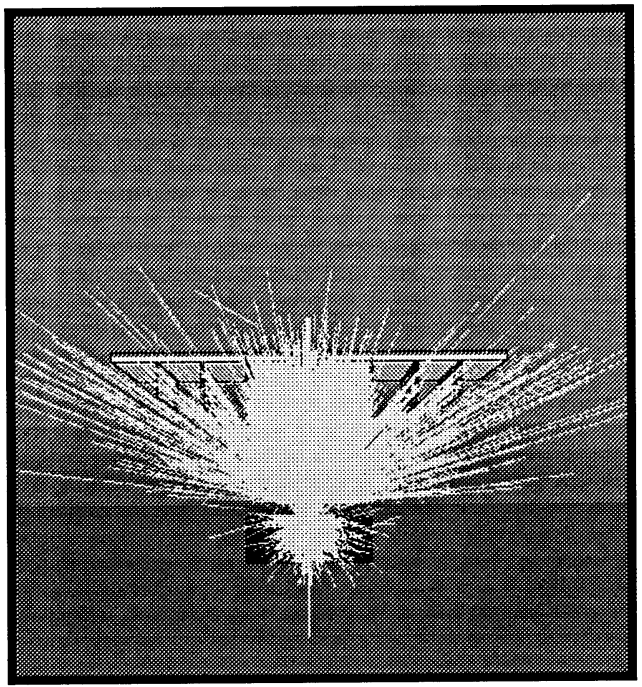

(a)

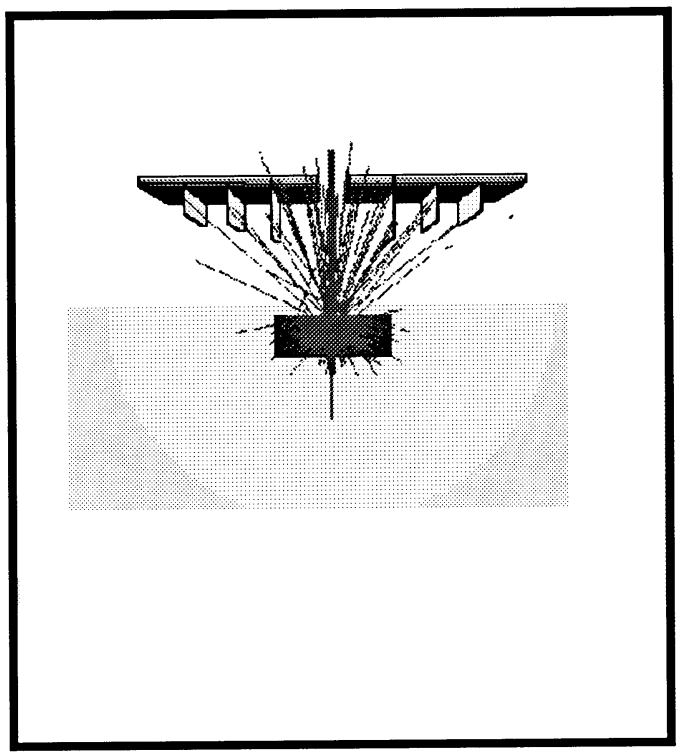

(b)

Fig. 9. The MCNP/SABRINA first-collision component tracks: (a) all first-collision components; (b) first-collision components from mine only. 


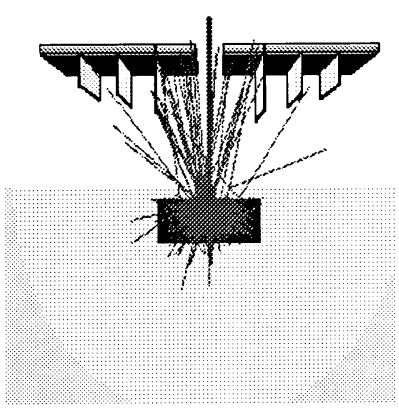

(a)

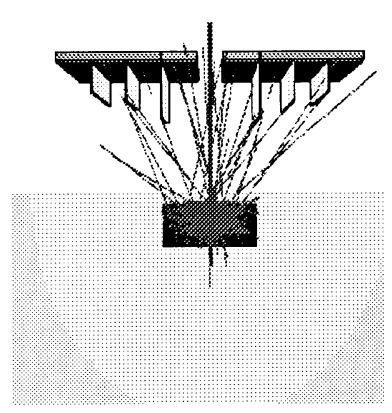

(b)

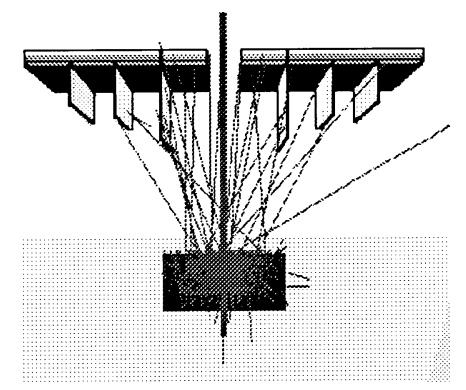

(c)

Fig. 10. The MCNP/SABRINA collision component tracks from the mine only: (a) second collision; (b) third collision; (c) fourth collision.

Table 1. The average scattering angles and energies of first scattered photons.

\begin{tabular}{|c|c|c|c|c|}
\hline $\begin{array}{l}\text { Energy } \\
(\mathrm{KeV})\end{array}$ & $\theta_{\mathrm{F}}$ & $\theta_{\mathrm{B}}$ & $E_{\mathrm{F}}$ & $E_{\mathrm{B}}$ \\
\hline 40 & 54.5 & 123.0 & 38.7 & 35.7 \\
\hline 60 & 53.9 & 122.4 & 57.3 & 50.9 \\
\hline 80 & 53.4 & 121.9 & 75.4 & 64.6 \\
\hline 100 & 52.9 & 121.4 & 93.0 & 77.2 \\
\hline 200 & 50.7 & 119.3 & 176.4 & 127.0 \\
\hline 300 & 49.1 & 117.6 & 253.5 & 162.8 \\
\hline 500 & 46.6 & 115.0 & 395.7 & 211.9 \\
\hline $1.00 E+03$ & 42.6 & 111.2 & 711.7 & 288.5 \\
\hline $5.00 E+03$ & 28.9 & 105.3 & 2832.1 & 383.5 \\
\hline
\end{tabular}


Table 2. Average scattering angles of backscattered components relative to the incident X-ray beam direction for $80-\mathrm{keV}$ photons in soil.

\begin{tabular}{|c|c|c|c|c|c|c|c|c|c|c|}
\hline $\begin{array}{c}\text { First } \\
\text { collision }\end{array}$ & \multicolumn{1}{|c|}{$\begin{array}{c}\text { Second } \\
\text { collision }\end{array}$} & \multicolumn{10}{|c|}{ Third collision } \\
\hline$\theta_{\mathrm{b}}$ & $\theta_{\mathrm{fb}}$ & $\theta_{\mathrm{bf}}$ & $\theta_{\mathrm{fff}}$ & $\theta_{\mathrm{ffb}}$ & $\theta_{\mathrm{fbf}}$ & $\theta_{\mathrm{fbb}}$ & $\theta_{\mathrm{bbf}}$ & $\theta_{\mathrm{bbb}}$ & $\theta_{\mathrm{bfb}}$ & $\theta_{\mathrm{bff}}$ \\
\hline 122 & 175 & 174 & 160 & -131 & -137 & 170 & -168 & 125 & -165 & -130 \\
\hline
\end{tabular}

f-Forward

b-Backward

40 and $100 \mathrm{keV}$, the average forward- and backscattering angles can be approximated as $53^{\circ}$ and $120^{\circ}$, respectively. The angular paths of first-, second-, and third-collision backscattered $80-\mathrm{keV}$ photons (i.e., those photons that move toward the detectors) based on average scattering angles are presented in Table 2 and Fig. 11. These calculated results show a behavior that is similar to the MCNP/SABRINA results.

The unique behavior of scattered photon collision components can be utilized to our advantage to design imaging detectors. The detectors used for LMR are plastic scintillator screens operating in the current mode, which enables us to measure the energy deposition in these detectors. These detectors are large-area detectors, and this helps not only in reducing the required source strength but also in averaging out the smaller background signal variations. The signal-to-noise ratio that defines the mine detectability in LMR images can be quantified by the mine-to-soil ratio $(M S R)$. The $M S R$ is given by the relationship $M S R=E_{m+s} / E_{s}$, where $E_{m+s}$ is the energy deposited in a detector in the presence of a mine and $E_{s}$ is the energy deposited in a detector in the absence of a mine (i.e., only from soil).

The principle design parameters that affect the performance of detectors are collimator lengths and raster gap size. For raster gap sizes from a few centimeters up to around $20 \mathrm{~cm}$, the effect of the gap size on the performance of the uncollimated detectors is small; however, because the gap size dictates the separation distance between collimated detectors, it also affects their performance. An increase in the raster gap results in an increase in separation between collimated detectors, which in turn causes a decrease in energy deposition in the collimated detectors. The size of the raster gap is limited by the required total energy deposition in the collimated detectors. Collimator length has a significant effect on the performance of collimated detectors. Using the MSR and total energy deposition in the detectors, the design of the collimated and uncollimated detectors can be optimized.

Table 3 presents the effect of collimator length on energy deposition and MSR by collision component for collimated detectors with a fixed raster gap. These results are generated through a series of MCNP simulations of the landmine detection system. Table 3 shows that the MSR increases with an increase in the collimator length up to an optimum value and decreases beyond this value. The first-collision component and total energy deposition decrease with an increase in the collimator length.

To visualize the effect of collimator length and raster gap size on the collision components, the photon transport in a landmine imaging system without collimators was simulated using MCNP/SABRINA, and the results are presented in Fig. 12. Figure 12a represents the 8609 first-collision tracks from 3890 histories. The second- and thirdcollision plots are presented in Fig. 12b and 12c, respectively. These figures represent 


\section{First Collision}

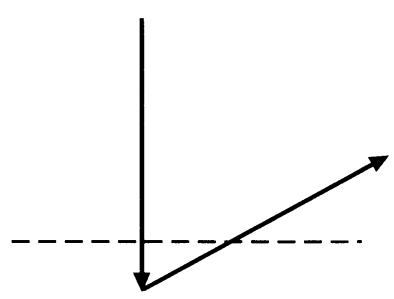

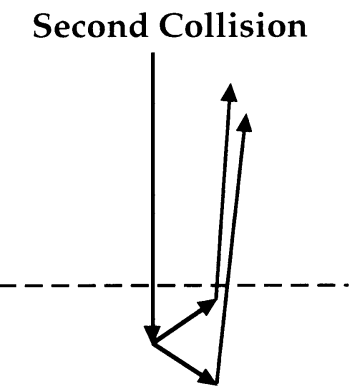

\section{Third Collision Component}
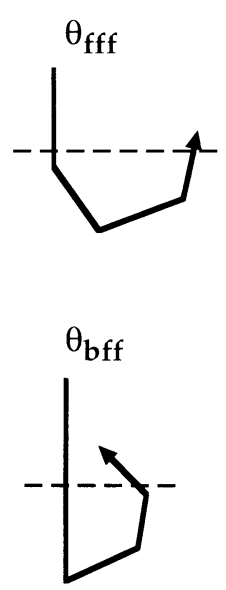
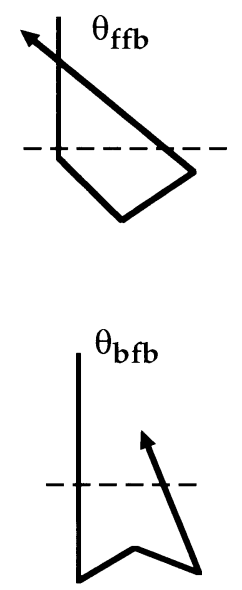
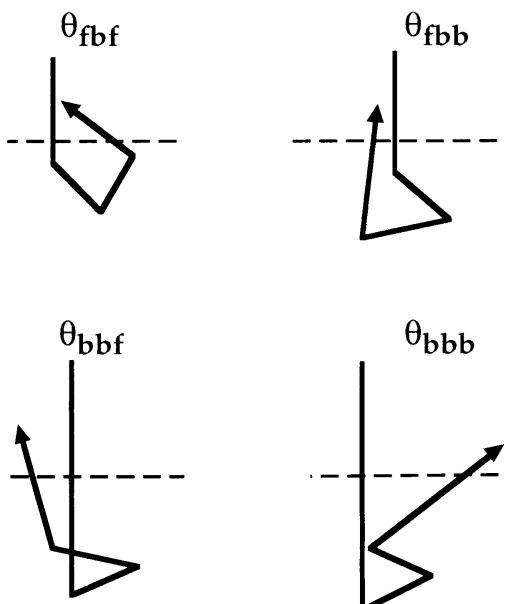

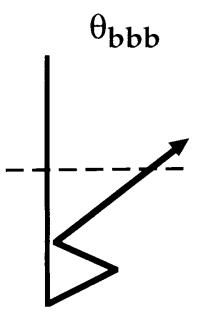

Fig. 11. Schematic representation of backscatter collision component paths using average scattering angles.

3877 histories/7949 tracks and 3886 histories/7508 tracks, respectively. The collimator shown in these plots was superimposed artificially on the track plots. It can be seen from these plots that the collimator with length shorter than the critical length is ineffective in stopping the surface-scattered photons; however, a collimator with length greater than the critical length eliminates not only scatters from the soil but also scatters from the mine. Additionally, collimators with larger than critical lengths remove not only the first scatter photons but also a significant number of higher collision photons. Thus, the MSR first increases with increasing collimator length and then decreases with an increase in the collimator length beyond the critical value. It can be seen from Fig. 9a and 12a that scatters from the surface do not show much lateral transport, and, hence, the collimator is more effective in reducing the signals from surface features in collimated detectors. From the MCNP simulations, the first collimator is found to be responsible for remov- 